1.2R Maastrup. 'Knowledge Centre for Breastfeeding Infants with Special Needs/NICU, Rigshospitalet, Copenhagen, Denmark; ${ }^{2}$ Department of Health Sciences, Faculty of Medicine, Lund University, Lund, Sweden

Background Breastfeeding of preterm infants is not well researched in Denmark, although breast milk is of great importance for these infants because it protects against infections, decrease the risk for severe diseases (necrotizing enterocolitis and retinopathy), and breastfeeding strengthens bonding and attachment between mother and infant. Many factors can influence breastfeeding of preterm infants. Some are related to the mother and infant, while some are related to unit-specific factors at the Neonatal Intensive Care Unit (NICU). This study will highlight potential influencing factors.

Aim To investigate how many preterm infants are breastfed exclusively at discharge, and breastfeeding duration. To examine which conditions in infants, mothers and NICUs that affect breastfeeding of preterm infants.

Method A multicenter exploratory longitudinal survey on breastfeeding of preterm infants in all NICUs in Denmark with questionnaires answered by the mothers in beginning of hospitalisation and at the infants' discharge, followed by telephone interviews in the infants' first year.

Preliminary results More than 1400 preterm infants from 24 to 36 weeks of gestational age (mean 33 weeks and 4 days, SD 249 weeks) participated in the study. At discharge $68 \%$ were exclusively breastfed, $17 \%$ were partially breastfed and $15 \%$ were not breastfed. Further analysis will show which factors in infants, mothers and NICUs influence breastfeeding at discharge.

Conclusion Compared to most international research, preterm infants in Denmark have high breastfeeding rates at discharge from NICU.

\section{THE EXPERIENCE OF NURSES TAKING CARE OF NEWBORNS WITH NEONATAL ABSTINENCE SYNDROME (NAS) AND THEIR FAMILIES}

doi:10.1136/archdischild-2012-302724.0067

'AP França, ${ }^{2}$ SM Pereira, 'MC Reisinho. 'UNIESEP (Oporto College of Nursing Resaearch Unit), Oporto College of Nursing, Porto; ${ }^{2}$ Unidade de Cuidados Especiais Pediátricos/ Neonatais, Hospital Santo Espírito de Angra do Heroísmo-EPER, Angra do Heroísmo, Portugal

This study entitled "The experience of nurses taking care of newborns with Neonatal Abstinence Syndrome (NAS) and their families" gives an account of the nurse's experiences on a Neonatal Intensive Care Unit (NICU), as well as the difficulties they face regarding this matter. This is a qualitative, exploratory and descriptive study, with a phenomenological approach. Data was collected by semi-structured interviews and submitted to content analysis (Bardin). The analyses brought out the following themes: how the nurse perceives the child and the parents he/she is taking care; how the nurse perceives the way that parents take care of their child; what the nurse feels when he/she is taking care of the child and the family; the difficulties the nurse has to face; what the nurse focuses his/her attention when taking care of the child and the family; what the nurse perceives as necessary to improve the care which is being provided.

Nurses expressed some difficulties concerning the teamwork, difficult relationship and communication with the families, and lack of resources, of a specific training and care standards, as well as an updated assessment tool. They feel frustration, anxiety, revolt, weariness, anguish, pain, as well as affection, satisfaction, challenge and recognition. In general, parents are absent and disinterested. However some are more present and provide basic care for their child autonomously.

This study has revealed the complexity and importance of taking care of such children, associated with their characteristics, clinical manifestations, suffering and peculiar characteristics of their families.

\section{PSYCHOLOGICAL SYMPTOMS OF ADOLESCENTS WITH AND WITHOUT CHRONIC ILLNESSES}

doi:10.1136/archdischild-2012-302724.0068

${ }^{1}$ A Ergun, ${ }^{2} S$ Topçu, ${ }^{2} S$ Albayrak, ${ }^{2} 1$ Isik. ${ }^{1}$ Community Health Nursing Department, Marmara University, Faculty of Health Science, Division of Nursing; ${ }^{2}$ Koc University, School of Nursing, Istanbul, Turkey

Background and aims The aim of this study was to compare psychological symptoms of adolescents with and without chronic illnesses.

Methods A total of 380 students ( 255 healthy and 85 with chronic illnesses) were recruited for the study. Data were gathered by using the general information form and the brief symptom inventory (BSI) for adolescent. Mann-Whitney-U test were used in analyzing the information.

Results Results demonstrated that adolescents with chronic illnesses had higher scores on somatization $(p<0.001)$ and lower scores on hostility $(p<0.05)$. Girls with chronic illnesses had lower scores on depression $(p<0.01)$, negative self-perception $(p<0.05)$ and hostility $(p<0.01)$ than healthy girls. Both girls and boys with chronic illnesses had higher somatization scores than peers without chronic illnesses.

Conclusions This research suggests the importance of mental health screening for adolescents with chronic illnesses and the need for clinical and community-based interventions to target modifiable lifestyle factors that contribute to psychological distress.

\section{FATIGUE IN CHILDREN BEFORE AND AFTER BONE MARROW TRANSPLANTATION: A PRELIMINARY DATA}

doi:10.1136/archdischild-2012-302724.0069

'F Petrangeli, 'F D'Agostino, 'A Sili, 2 T Petrangeli, 'S Alessandrini, 'R Alvaro. 'School of Nursing, University 'Tor Vergata'; 'Department of Pediatric Hematology and Oncology, Bambino Gesù Children's Hospital, IRCCS, Rome, Italy

Background and aims Fatigue is described as one of the most frequent and distressing symptoms of cancer therapy but in children and adolescents it has received limited clinical attention. The aim of the study was to assess the change in fatigue scores during cancer treatment according to children's, adolescents' and parents' perspectives and to describe the possible causes of fatigue.

Methods In this longitudinal descriptive study were enrolled 21 patients (aged8-18 years) 15(71\%) boys and 6(29\%) girls, with acute lymphoblastic (ALL66.6\%) and myeloid (AML33.4\%) leukemia, and one of their parents. Fatigue as measured by PedsOL Multidimensional Fatigue Scale the day before the first cycle of chemotherapy (T0) and after 30 days (T1) of induction chemotherapy (about 25 days after BMT).

Results Compared data pre and post chemotherapy children had significantly worse fatigue total scores (79.3 DS $\pm 10.5 \mathrm{vs} 42.9 \mathrm{DS} \pm 8.5$, $\mathrm{p}=0.000)$ : general fatigue domain (77.4DS $\pm 14.4 \mathrm{vs} 31.3 \mathrm{DS} \pm 10.1$ $\mathrm{p}=0.000$ ), sleep/rest fatigue domain $(73.4 \mathrm{DS} \pm 15.8 \mathrm{vs} 28.6 \mathrm{DS} \pm 12.1$, $\mathrm{p}=0.000$ ), and cognitive fatigue was the better and similar domain pre and after BMT in child's (87.1DS \pm 11.2 vs69DS $\pm 17.5, p=0.000$ ) and parents' (88.3DS+12vs68.2DS \pm 21.4 ) perception. Children with ALL (41.7DS \pm 8.9 ) had a worse fatigue than AML (45.6DS \pm 7.6 ) and the fatigue parent's perception about their children is worse than child's perception (38.2vs42.9).

Conclusions This study shown that cancer treatment increase significantly children's fatigue levels. Nurses should frequently assess fatigue in children receiving chemotherapy and apply timely and tailored interventions to match the factors that contribute to fatigue and influence fatigue severity, further to identify the domains compromises and make correct nursing diagnoses. Management of fatigue during treatment will help children stay involved in age-related activities and meet developmental milestones. 
70 PAIN EXPERIENCED DURING VENIPUNCTURE IS HIGHER IN CHILDREN WITH A CHRONIC DISEASE

doi:10.1136/archdischild-2012-302724.0070

F Festini, C Dini, S Bisogni. University of Florence, Florence, Italy

Background Venipuncture pain in children results from several factors, which amplify the nociceptive stimulus; among them anticipatory anxiety plays an important role. Children with a chronic disease undergo invasive procedures and venipunture more frequently than other children.

Goal To evaluate whether a difference exists in pain threshold between children with a chronic disease and children with no previous experience of procedural pain.

Methods Cross-sectional analytical trial. A group of children with a chronic disease (group A) and a group of children with no previous experience of venipuncture (group B) aged 4 to 12 were observed during a standardized venipuncture for blood sample drawing. Pain was self-rated with a 1-10 Wong faces scale or a 1-10 numeric scale. Behavioural distress was measured with the Observational Behaviour Distress Scale (OBDS, 1-33).

Results 230 children were examined, 82 in group A and 148 in group $B$. With regards to pain, children in group A reported a mean pain score of 8.6 ( $\mathrm{sd} 1.2)$ while children in group B had a mean score of 3.1 (sd 2.4), Anova $p=0.00001$. With regards to behavioural distress, children in group A had a mean score of 26.7 (sd 4.3) vs. 8.3 in group B (sd 8.4), Anova $\mathrm{p}=0.00001$. The groups did not show statistically significant differences in age and sex distribution.

Discussion Children with a chronic disease, who previously underwent numerous venipunctures, experience a more intense pain and distress than their peers at their first experience of venipuncture.

\section{RELIABILITY AND VALIDITY OF TURKISH VERSION OF THE PHYSICAL ACTIVITY OUESTIONNAIRE FOR OLDER CHILDREN (PAQ-C)}

doi:10.1136/archdischild-2012-302724.0071

'L Erdim, ${ }^{2} \mathrm{~A}$ Ergun, ${ }^{3} \mathrm{~S}$ Kuguoglu. ${ }^{1}$ Pediatric Nursing Department, Istanbul University, Faculty of Health Science; ${ }^{2}$ Community Health Nursing, Marmara University, Faculty of Health Science, Division of Nursing, Istanbul, Turkey; ${ }^{3}$ Pediatric Nursing Department, Emeritus Professor from Marmara University, Faculty of Health Science, Division of Nursing, Brooklyn, NY, USA

Background and aims The aim of this study was to examine the psychometric properties of Turkish version of the Physical Activity Questionnaire for Older Children (PAQ-C) developed by Kowalski et al. The PAQ-C is a validated self-report measure of physical activity widely used to assess physical activity in children (8-14 years of age).

Methods This study was methodological design. The PAQ-C consists of nine questions was rated on a 5-point likert scale. The $\mathrm{PAQ}-\mathrm{C}$ was translated using a translation/back-translation method. The subjects were 549 Turkish school children. The internal consistency of the PAQ-C was assessed using Cronbach's alpha. Content validity was based on an experts' panel and assessed by using a content validity index

Results The study was completed with 549 children $(49.8 \%$ female, $50.2 \%$ male). Mean age of the participant was 10.30 years $(\mathrm{SD}=0.71$, range, $8-11$ years). The overall Content validity index (CVI) was $98.75 \%$ which signified that the PAQ-C has good content validity. Correlation values of scale items were found between $0.21-$ 0.70 for all items as the result of item total score analysis. Cronbach's alpha coefficient was found as 0.86 . The scale had a fairly good test-retest reliability $(r=0.64, \mathrm{p}<0.001)$.

Conclusions Psychometric analyses of the Turkish version of PAO-C indicate high reliability and good content and construct validity. The PAQ-C is a useful instrument for assess to physical activity patterns in Turkish children.

\section{INCIDENCE AND OUTCOME OF INFLICTED TRAUMATIC BRAIN INJURY AT THE PAEDIATRIC INTENSIVE CARE UNITS IN THE NETHERLANDS}

doi:10.1136/archdischild-2012-302724.0072

${ }^{1} \mathrm{~J}$ Elias, ${ }^{2} \mathrm{~N}$ Jansen, ${ }^{3 \mathrm{P}}$ van Hasselt. ${ }^{\mathrm{U}} \mathrm{UMC}$ Utrecht; ${ }^{2}$ Department of Pediatric Intensive Care; ${ }^{3}$ Department of Pediatric Metabolic Diseases, Wilhelmina Children's Hospital, Utrecht, The Netherlands

Background Inflicted traumatic brain injury (iTBI) is recognized as a major cause of death and poor outcome in young children.

Aim To determine the incidence and outcome of children with iTBI at the Paediatric Intensive Care Units (PICUs) in the Netherlands.

Methods The Dutch PICU registry was used to identify all children (0-2 years) who were admitted with traumatic brain injury (TBI) between 1-1-2005 and 31-12-2009. ITBI was defined as (1) proven with a confession or conviction, (2)confirmed by the Child protective services or (3)brain injuries, fractures or retinal hemorrhages incompatible with, or without history of trauma. Cases of iTBI were used to calculate the incidence for each year. Outcome parameters were compared for children with iTBI versus non-iTBI. Results During the study period 70 of 166 had iTBI (42\%), resulting in an overall incidence of 7.6/100.000 live births. The single year incidence varied markedly between 3.3 and 12.4 per 100.000 . The children with iTBI were younger than the children with non-iTBI (4.36 versus 7.49 months, $p<0.001$ ). Children with iTBI stayed significantly longer at the PICU (7.10 versus 3.92 days, $\mathrm{p}<0.01)$ and in hospital (27.62 versus 7.59 days, $p<0.001)$, and were significantly longer on mechanical ventilation (4.23 versus 1.65 days, $p<0.01$ ) iTBI was associated with a significantly higher mortality rate as compared with non-iTBI ( $23 \%$ vs $2 \%$, p<0.001).

Conclusions Of young children admitted to Dutch PICUs with TBI $42 \%$ had iTBI. The overall incidence of iTBI was 7.6/100.00. The children with iTBI had a significantly higher mortality rate of almost 25\%.

\section{HYPOTHERMIA-TREATED INFANTS WITH HYPOXIC- ISCHEMIC ENCEPHALOPATHY (HIE): MRI-FINDINGS CORRELATE WELL WITH NEUROMOTOR-OUTCOME AT 12 MONTHS}

doi:10.1136/archdischild-2012-302724.0073

${ }^{1} \mathrm{~K}$ Robertson Grossmann, ${ }^{2} \mathrm{~A}$ Tzovla, ${ }^{2} \mathrm{M}$ Kristoffersen Wiberg, ${ }^{1} \mathrm{~B}$ Hallberg. ${ }^{\prime}$ Neonatology; ${ }^{2}$ Radiology, Karolinska Institute, Karolinska University Hospital, Stockholm, Sweden

Background Induced hypothermia treatment (HT) for 72 hours reduces the risk of neurological sequele in asphyxiated full-term infants. Pathological findings on postnatal MRI of the brain have been shown to correlate to the prognosis.

This population-based study investigates the correlation between pathological findings in MRI of the brain after HT and neuromotor outcome at 12 months of age in asphyxiated full-term infants with HIE.

Methods In Stockholm, between January 2006 and December 2009, all infants fulfilling A- and B-criteria for HT were included. MRI of the brain was done within 2 weeks from birth. Neurological assessment was done at 4 and 12 months of age.

Results Of the 70 infants who received HT, both MRI examination and outcome data were available for 60 infants. Patterns of injury on MRI were defined based on the predominant site of injury: Watershed predominant (WS), basal ganglia/thalamus predominant $(\mathrm{BG} / \mathrm{T})$, general/global lesions $(\mathrm{G})$ and normal. 